\title{
Sar1 Forms Ordered Arrays That Can Facilitate Vesicle Scission in the COPII Pathway.
}

\author{
Hanaa Hariri ${ }^{1}$, Alex Noble ${ }^{2}$, Arthur Omran ${ }^{3}$, and Scott M. Stagg ${ }^{1,2}$ \\ 1. Institute of Molecular Biophysics, Florida State University, Tallahassee, FL, USA \\ 2. Department of Physics, Florida State University, Tallahassee, FL, USA \\ 3. Department of Chemistry and Biochemistry, Florida State University, Tallahassee, FL, USA
}

Secretion is a fundamental process in eukaryotes. It's disruption can lead to one of several diseases including chylomicron retention disease ${ }^{1}$, cranio-lenticulo-sutural dysplasia ${ }^{2}$, or congenital dyserythropoietic anemia ${ }^{3}$. Transport of secreted cargo such as membrane proteins or soluble secreted cargos is initiated at the endoplasmic reticulum (ER). There proteins are co-translationally translocated into the lumen of the ER. These cargo proteins are then concentrated at specific sites on the ER called exit sites, and are collected into a membrane-bound vesicle that is transported to the Golgi apparatus and on to the cell surface. The coat protein complex II (COPII) proteins Sar1, Sec23/24, and Sec13/31 facilitate the formation of transport vesicles at the ER exit sites ${ }^{4}$. Vesicle formation is completed when the vesicle detaches from the parent membrane in a process called scission. It has been shown that Sar1 is required for scission ${ }^{5}$ though the mechanism by which the bud neck is collapsed and fused remains unclear.

We have shown that Sar1 can oligomerize on membranes and that it can vesiculate giant unilamellar vesicles (GUVs) in the absence of the other COPII proteins. It is clear that there is not enough Sar1 expressed in cells for it to facilitate vesiculation on its own; instead it works in concert with the other COPII proteins to form vesicles in the right place at the right time. We have hypothesized that the oligomerization of Sar1 plays two roles in COPII mediated vesiculation: 1) to initiate membrane curvature, and 2) to help facilitate scission.

Here we show that Sar1 can transform membranes into a variety of stuctures including sheets, multibudded vesicles that are connected by narrow necks, and coated tubules with diameters ranging from $45 \mathrm{~nm}$ too $200 \mathrm{~nm}$ (Fig. 3). A low-resolution structure of the Sar1 lattice was determined by limiting the diameter of the tubules using galactosyl ceramide lipids and using iterative helical reconstruction. This revealed that the Sar1 lattice consists of arrays of dimers, but the resolution was too low to be able to determine the residues that mediate the intermolecular contacts in the lattice or to resolve how the N-terminal amphipathic is buried in the outer membrane leaflet to anchor Sar1 to the membrane. We are currently characterizing the mechanisms by which Sar1 deforms membrane by determining what residues mediate the formation of the Sar1 lattice and by determining how Sar1 inserts into the bilayer. We show that Sar1 forms ordered arrays on membranes with low curvature, and with more highly curved membranes, it only forms locally ordered arrays (Fig. 1), and we propose a mechanism by which Sar1 facilitates vesicle scission at COPII bud necks by polymerization of Sar1 dimers. 
References:

1. Shoulders C. C., Stephens D. J. \& Jones B. (2004). The intracellular transport of chylomicrons requires the small GTPase, Sar1b. Curr Opin Lipidol 15, 191-197.

2. Boyadjiev S. A., Fromme J. C., Ben J., Chong S. S., Nauta C., Hur D. J., Zhang G., Hamamoto S., Schekman R., Ravazzola M., Orci L. \& Eyaid W. (2006). Cranio-lenticulo-sutural dysplasia is caused by a SEC23A mutation leading to abnormal endoplasmic-reticulum-to-Golgi trafficking. Nat Genet 38, $1192-1197$.

3. Schwarz K., Iolascon A., Verissimo F., Trede N. S., Horsley W., Chen W., Paw B. H., Hopfner K. P., Holzmann K., Russo R., Esposito M. R., Spano D., De Falco L., Heinrich K., Joggerst B., Rojewski M. T., Perrotta S., Denecke J., Pannicke U., Delaunay J., Pepperkok R. \& Heimpel H. (2009). Mutations affecting the secretory COPII coat component SEC23B cause congenital dyserythropoietic anemia type II. Nat Genet 41, 936-940.

4. D'Arcangelo J. G., Stahmer K. R. \& Miller E. A. (2013). Vesicle-mediated export from the ER: COPII coat function and regulation. Biochim Biophys Acta 1833, 2464-2472.

5. Lee M. C., Orci L., Hamamoto S., Futai E., Ravazzola M. \& Schekman R. (2005). Sar1p N-terminal helix initiates membrane curvature and completes the fission of a COPII vesicle. Cell 122, 605-617.

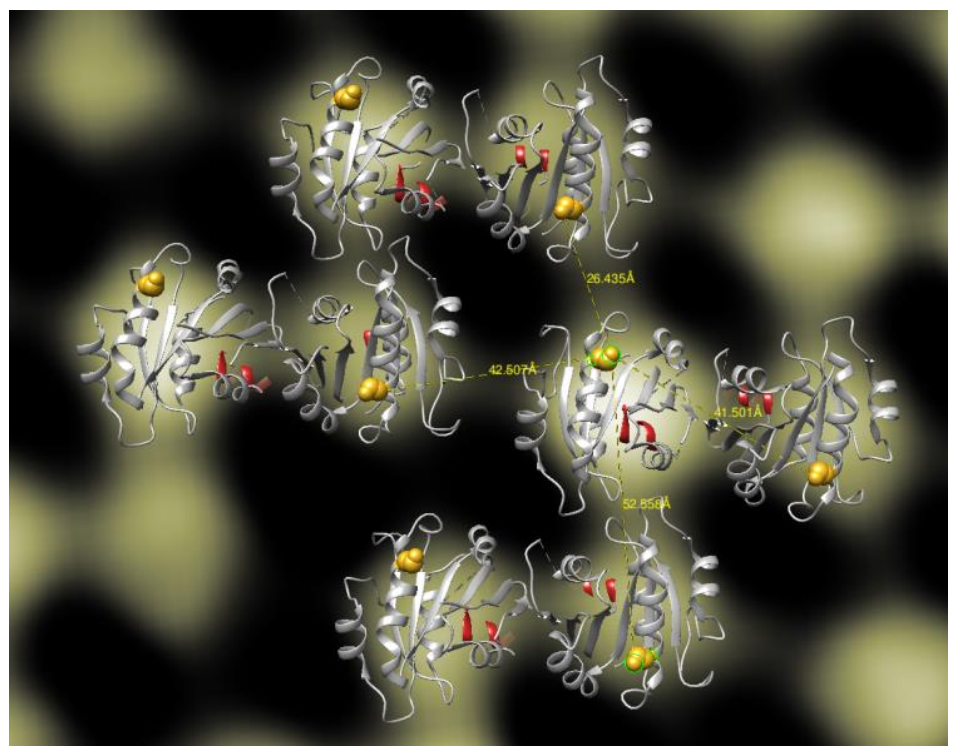

Figure 1. Structure of the lattice of Sar1 dimers. Sar1 self assembles into a 2D lattice on membranes with low curvature. Class averages (yellow) indicated that the basic assembly unit of the Sar1 lattice is a dimer. 